\title{
Personenmerkmale und Kommissionierleistungen bei Verwendung eines Pick-by-Watch-Assistenzsystems oder herkömmlicher Papierlisten
}

\author{
Torsten J. Gerpott • Ahmet Kurt
}

Eingegangen: 28. Mai 2021 / Angenommen: 13. Juli 2021 / Online publiziert: 3. August 2021

(C) Der/die Autor(en) 2021

Zusammenfassung In der Kommissionierung werden anstelle von Papierlisten (Pick-by-Paper) zunehmend digitale Assistenzsysteme (AS) eingesetzt. Hierzu gehören vernetzte Armbanduhren zur Weitergabe von Auftragsdaten an das Personal während es sich im Lager bewegt, um Produkte zu entnehmen (Pick-by-Watch $[\mathrm{PbW}])$. Bislang hat die Forschung sich auf die ergonomische und technische Gestaltung von $\mathrm{PbW}$-Systemen sowie ihre Leistung relativ zu anderen AS konzentriert. Kaum analysiert wurde, ob individuelle Merkmale von Kommissionierern (z. B. Alter) systematische Zusammenhänge mit Leistungsaspekten von AS aufweisen und solche Assoziationen bei verschiedenen AS voneinander abweichen. Ziel der vorliegenden Studie ist es deshalb, Korrelationen zwischen individuellen Merkmalen von Kommissionierern einerseits und Kommissionierleistungskriterien andererseits bei Verwendung eines $\mathrm{PbW}$-Systems oder herkömmlicher Papierlisten empirisch zu bestimmen. Hierzu wurde ein Laborexperiment mit 55 Teilnehmern durchgeführt, für die fünf Dimensionen von Kommissionierleistungen jeweils für ein $\mathrm{PbW}$ AS sowie Papierlisten und fünf personenbezogene Merkmale gemessen wurden. Die empirischen Befunde erweitern den Kenntnisstand zu Spielräumen und Grenzen der Leistungsbeeinflussung in der Kommissionierung durch Einarbeitungs- und Auswahlmaßnahmen, die auf soziodemographische und tätigkeitsbezogene Erfahrungsmerkmale der Aufgabenträger Bezug nehmen.

Schlüsselwörter Digitale Assistenzsysteme · IT-Unterstützung ·

Kommissionierung · Leistungsmessung · Papierlisten · Smart Watches

\footnotetext{
Torsten J. Gerpott $(\bowtie) \cdot$ Ahmet Kurt

Lehrstuhl Unternehmens- und Technologieplanung, Mercator School of Management, Universität

Duisburg-Essen, Lotharstr. 65, 47057 Duisburg, Deutschland

E-Mail: torsten.gerpott@uni-due.de

Ahmet Kurt

E-Mail: ahmet.kurt@uni-due.de
} 


\title{
Personal Characteristics and Commissioning Performance when Using a Pick-by-Watch Assistance System or Conventional Paper Lists
}

\begin{abstract}
Digital assistance systems (AS) are increasingly used to replace paper lists in commissioning processes. Such systems include wristwatches transmitting order data to pickers moving through a storehouse to collect products (pick-bywatch $[\mathrm{PBW}])$. Prior scholarly work has focused on ergonomic and technical design issues of PBW systems and their performance relative to other AS. In contrast, there is a research lacuna on whether personal characteristics of pickers (e.g., age) are systematically associated with performance dimensions of AS and on whether such associations vary across different AS. Therefore, the objective of the present study is to empirically explore correlations between individual characteristics of pickers and commissioning performance criteria in case that either a PBW system or a paper list is supporting the process. For this purpose, a lab experiment with 55 participants was conducted to measure five commissioning performance dimensions both for a PBW-AS and paper lists on the one hand and five personal characteristics of pickers on the other. The empirical findings contribute to the state of knowledge on margins for manoeuvre of induction and selection measures targeting socio-demographic and task-related experience characteristics of pickers to influence individual commissioning performance.
\end{abstract}

Keywords Commissioning $\cdot$ Digital order picking assistance systems $\cdot$ IT support $\cdot$ Performance measurement $\cdot$ Paper lists $\cdot$ Smart Watches

\section{Einordnung der Untersuchung}

Die manuelle Kommissionierung spielt bei der Gestaltung von Lagerabläufen eine wichtige Rolle, da sie sich durch eine hohe Personal- sowie eine daraus resultierende hohe Kostenintensität auszeichnet (Ponis et al. 2020, S. 1621; Ten Hompel et al. 2019, S. 113; Tripp 2019, S. 2-3; Van Gils et al. 2018, S. 1). Gemäß VDI (1994, S. 2) umfasst diese Tätigkeit die auftragsbezogene Zusammenstellung einer Teilmenge materieller Güter aus dem Gesamtbestand eines Lagers. In der Vergangenheit wurden bei der Kommissionierung Papierlisten (Pick-by-Paper (PbP)) eingesetzt, um Fehler zu reduzieren und die Geschwindigkeit zu steigern. Seit etwa zehn Jahren werden zunehmend informatische Assistenzsysteme (AS) in Kommissionierprozesse eingebunden, um Mitarbeiter zu unterstützen (vgl. Egbert et al. 2020, S. 74-75; Franzke 2018, S. 38). Zu solchen Geräten gehören vernetzte Armbanduhren mit berührungsempfindlichen Bildschirmen (Smart Watches), die unter dem Oberbegriff Pick-by-Watch (PbW) Systeme zusammengefasst werden. Sie teilen auf eine vom Kommissionierer getragene Uhr Auftragsinformationen mit, die von einem Leitstand unter Rückgriff auf eine programmierbare Schnittstelle und eine funkgestützte Telekommunikationsverbindung disponiert werden.

In der wissenschaftlichen Literatur wurden bislang primär ergonomische und technische Gestaltungsfragen von $\mathrm{PbW}$-Systemen sowie ihre Leistung relativ zu anderen AS (z. B. Datenbrillen/Smart Glasses, Pick-by-Light) untersucht (vgl. Gerpott 
und Kurt 2020). Kaum betrachtet wurde demgegenüber, ob Merkmale von Kommissionierern (z.B. Alter) systematische Zusammenhänge mit Leistungsaspekten von AS aufweisen (s. als Ausnahme de Vries et al. 2016) und solche Assoziationen bei verschiedenen AS voneinander abweichen. Sollten derartige Korrelationen bestehen, dann ergibt sich die Möglichkeit, Mitarbeiter mit bestimmten Merkmalsprofilen gezielt mit einem AS (nicht) auszustatten oder kompensatorisch bei Mitarbeitern, die tendenziell Probleme mit einem AS haben (z. B. Schwierigkeiten beim Ablesen von Auftragsinformationen), besondere Qualifizierungsschritte zu ergreifen (vgl. Werning et al. 2019).

Ziel der vorliegenden empirischen Studie ist es deshalb, Korrelationen zwischen individuellen Merkmalen von Kommissionierern einerseits und Kommissionierleistungskriterien andererseits bei Verwendung eines $\mathrm{PbW}$-Systems oder herkömmlicher Papierlisten zu analysieren. Hierzu wurde ein Laborexperiment mit 55 Teilnehmern durchgeführt, für die fünf Dimensionen von Kommissionierleistungen jeweils für ein $\mathrm{PbW}-\mathrm{AS}$ sowie Papierlisten und fünf personenbezogene Merkmale gemessen wurden.

Im Folgenden konkretisiert Abschn. 2 die eigenen Untersuchungshypothesen. Abschn. 3 stellt die Methodik der empirischen Untersuchung vor, deren statistische Auswertung in Abschn. 4 präsentiert wird. Abschließend werden Implikationen für Praxis und Wissenschaft aus den Ergebnissen in Abschn. 5 abgeleitet.

\section{Untersuchungshypothesen}

Papierlisten sind die älteste Variante zur Zusammenstellung der für einen Auftrag zu entnehmenden Artikel. Der Kommissionier führt einen Papierbogen mit den benötigten Informationen mit sich und hat so bei der Entnahme von Artikeln nur eine Hand frei. Zusätzlich kann ein Klemmbrett genutzt werden, das mit einem Stift versehen ist, um bereits entnommene Positionen auf der Kommissionierliste zu markieren.

Bei Smart Watches in der Kommissionierung erscheinen per Funknetz (zumeist WLAN) übertragene Aufträge auf einer Armbanduhr mit Telekommunikationsmodul in Form einzelner Entnahmepositionen. Die jeweiligen Entnahmemengen erschließt sich der Kommissionierer durch eine Wischfunktion auf der Anzeigefläche der Smart Watch. Die Richtigkeit der Entnahmestelle kann vor der Anzeige der Entnahmemenge mit einem einzigartigen Code geprüft werden. Zur Reduktion von Ablesefehlern werden für industrielle Anwendungen oft speziell gefertigte Uhren mit großem Display, automatischem Zoom und starker Helligkeit eingesetzt. Des Weiteren kann man auf solchen Uhren zusätzliche Funktionen wie haptische Signale (z.B. Vibration) bei Fehleingaben, bestimmten Prozessschritten oder Erreichen von Gefahrenstellen implementieren. Smart Watches ermöglichen eine freihändige Auftragsbearbeitung. Gegenüber Smart Glasses als AS in Logistik, Produktion oder Kundendienst (vgl. Werning et al. 2019; Berkemeier et al. 2017) erfordern Smart Watches niedrigere Geräteinvestitionen. Im Vergleich zu mobilen Scannern sind Smart Watches leicht und am Handgelenk fixiert, so dass gewährleistet ist, dass das AS nicht abgelegt werden kann und folglich auch nicht zeitaufwändig wiedergefunden/-aufgenommen werden muss. 
Papierlisten und $\mathrm{PbW}$-AS unterscheiden sich bezüglich der Art der Informationsaufnahme und -abgabe. PbW-AS stellen typischerweise komplexere Anforderungen an die visuelle und haptische Informationsaufnahme als Papierlisten, da auf Smart Watches Auftragsdaten kleiner dargestellt und z. T. haptisch vermittelt werden. Zudem sind bei Uhren motorisch schwierigere Rückmeldungen über gezielte Berührungen notwendig als bei Papierlisten (vgl. Schlick et al. 2018, S. 432 u. 445-446). Papierlisten können höhere Gedächtnisleistungen bedingen als PbW-Systeme, sofern eine Auftragsposition eine beidhändige Entnahme auslöst und deshalb die Liste aus der Hand gelegt wird. Papierlisten und $\mathrm{PbW}$-AS sollten sich allerdings nicht divergierend auf die Beweglichkeit von Kommissionierern im Lager auswirken, da nicht ersichtlich ist, dass eines der mitgeführten Hilfsmittel infolge höheren Gewichts oder unhandlicherer Abmessungen stärkere negative Effekte auf die Bewegungsgeschwindigkeit haben dürfte.

Bislang ist aufgrund fehlender empirischer Daten unklar, inwiefern Kommissionierleistungen bei Einsatz von $\mathrm{PbW}$ - und $\mathrm{PbP}$-AS auch eine Funktion soziodemographischer Merkmale und vom Erfahrungsaspekten der Mitarbeiter sind (vgl. Grosse et al. 2015, S. 695-696). Ebenso wurde nicht analysiert, inwieweit die umrissenen Anforderungsunterschiede zwischen $\mathrm{PbW}$-AS und Papierlisten dazu beitragen, dass auch Mitarbeitermerkmale bei PbW-AS anders mit Kriterien von Kommissionierleistungen korrelieren als bei Papierlisten. Praktische Relevanz haben hier vor allem die fünf Merkmale Alter, Geschlecht, Nationalität, berufliche Kommissioniererfahrungen und generelle Nutzungserfahrungen mit Smart Watches, weil sie einfach zu ermitteln sind oder direkte Bezüge zu den betrachteten Tätigkeiten bzw. AS haben.

Bezüglich des Alters sprechen Meta-Analysen der empirischen Forschung (s. Rhodes et al. 2019; Tucker-Drob et al. 2019) dafür, dass eine negative Korrelation des Alters mit Fähigkeiten zur visuellen und haptischen Informationsaufnahme, die bei PbW-AS mehr gefordert werden als bei Papierlisten, sowie der Bewegungsgeschwindigkeit zu vermuten ist. Deshalb testen wir als Hypothesen $(\mathrm{H}) 1$ und 2:

$\mathbf{H}_{1}$ Das Alter eines Kommissionierers korreliert sowohl bei Einsatz eines PbWAS als auch bei Verwendung von Papierlisten signifikant negativ mit Kriterien der Kommissionierleistung.

$\mathbf{H}_{2}$ Korrelationen zwischen Alter und Kommissionierleistungsdimensionen sind bei einem $\mathrm{PbW}$-AS stärker negativ als bei Papierlisten.

Im Hinblick auf das Merkmal Geschlecht ist empirisch vielfach belegt (s. Siddiq und Scherer 2019; Hyde 2005, 2014), dass Männer und Frauen sich nicht signifikant in ihren für Kommissionieraufgaben relevanten Informationsaufnahme- und -abgabefähigkeiten sowie mäßigen (Geh-)Geschwindigkeiten unterscheiden. Somit formulieren wir:

$\mathbf{H}_{3}$ Das Geschlecht eines Kommissionierers korreliert sowohl bei Einsatz eines $\mathrm{PbW}-\mathrm{AS}$ als auch bei Verwendung von Papierlisten nicht signifikant mit Dimensionen der Kommissionierleistung. 
Das Merkmal Nationalität kann als Indikator für die Fähigkeit, in deutscher Sprache schriftliche Informationen aufzunehmen (Lesekompetenz), interpretiert werden. Personen mit deutscher Nationalität weisen im Durchschnitt aufgrund ihrer Sozialisation durch Familie und privates sowie berufliches Umfeld bei dieser Fähigkeit signifikant höhere Werte auf als Personen mit anderer Nationalität bzw. Migrationshintergrund (vgl. Diehl et al. 2016; Esser 2008; Gogolin et al. 2021; Schunck und Teltemann 2019; Seeber et al. 2019). Der effektive Einsatz von PbW-AS und Papierlisten setzen höhere Ausprägungen dieser Fähigkeit voraus. Als Konsequenz ergibt sich:

$\mathbf{H}_{4}$ Die Nationalität eines Kommissionierers korreliert signifikant mit Dimensionen der Kommissionierleistung in dem Sinne, dass sowohl bei Einsatz eines PbW-AS als auch bei Verwendung von Papierlisten Mitarbeiter mit deutscher Nationalität bessere Leistungsausprägungen aufweisen als Mitarbeiter ohne deutsche Nationalität.

Die Forschung zu Trainingseffekten bei manuellen Kommissionierungsprozessen (s. Grosse und Glock 2013, 2015) stützt die Vermutung, dass Erfahrungen im Umgang mit Kommissionieraufgaben im allgemeinen sowie kontextübergreifende Nutzungserfahrungen mit Smart Watches weitere Mitarbeitermerkmale darstellen, die sich auf Kommissionierleistungen positiv auswirken. Entsprechend überprüfen wir:

H5 $_{5}$ Die Kommissioniererfahrung eines Mitarbeiters korreliert sowohl bei Einsatz eines $\mathrm{PbW}$-AS als auch bei Verwendung von Papierlisten signifikant positiv mit Dimensionen der Kommissionierleistung.

$\mathbf{H}_{6}$ Die Nutzungserfahrung eines Kommissionierers mit Smart Watches korreliert (a) bei einem PbW-AS signifikant positiv und (b) bei Papierlisten nicht signifikant mit Dimensionen der Kommissionierleistung.

\section{Empirische Methodik}

\subsection{Lageraufbau und Assistenzsysteme}

Für die Untersuchung wurde ein experimentelles Lager mit vier alphabetisch gekennzeichneten Regalzeilen (Länge: 4,00 m, Tiefe: 0,25 m), fünf Ebenen (Höhe Oberbzw. Untergrenze der bestückten Regalebenen: 1,57 m bzw. 0,52 m) und zwei Gängen (Breite: 1,16m) errichtet. Auf jeder Ebene wurden vier Böden mit jeweils zwei Plätzen für Lagergüter montiert. Für kleinere Lagergüter wurden die Regalböden mit bis zu zwei Sichtlagerkästen besetzt. Größere Artikel, wie z.B. Kopierpapier oder Ordner, wurden ohne Kasten auf einem Regalboden platziert. Insgesamt wurden so 96 Lagerplätze erzeugt ( 4 Zeilen $\times 3$ Ebenen $\times 4 \times$ Böden $\times 2$ Orte), die mit verschiedenen Gütern gefüllt und mit den Merkmalen Regalzeile, Regalebene und Fachnummer beschriftet wurden. 
In dem Lager erhielten die Versuchsteilnehmer insgesamt zehn Kommissionieraufträge aus verschiedenen Artikeln mit unterschiedlichen Entnahmemengen, von denen eine Hälfte mit Papierlisten und die andere mit einem $\mathrm{PbW}$-AS bearbeitet wurde. Die Zahl der Artikel ( $\geq 3$ und $\leq 5$ ) sowie die Entnahmemengen $(\geq 1$ und $\leq 20)$ wurden zufällig erzeugt. Das zuerst eingesetzte Kommisionierverfahren wurde alternierend variiert, so dass 50\% der Probanden mit dem PbP- (PbW-)System starteten und danach das $\mathrm{PbW}-(\mathrm{PbP}-) \mathrm{AS}$ verwendeten. Systemübergreifend erhielten die Teilnehmer einen Schiebewagen zum Ablegen der Artikel eines Auftrags. Wegfolgen wurden nicht vorgegeben. Vor Beginn eines Auftrags wurde der Wagen am selben Startort positioniert. Ein Auftrag wurde als beendet eingestuft, sobald der Kommissionierer nach Entnahme der Artikel mit dem Wagen wieder die Startposition erreicht hatte.

Jeder Kommissionierer durchlief sechs Ablaufschritte, die pro Person insgesamt etwa eine Stunde Zeit in Anspruch nahmen:

1. Lektüre Teilnahmeinformationen und Unterzeichnung Einverständniserklärung

2. Erläuterung Lageraufbau und Versuchsablauf (inklusive Demonstration von drei Aufträgen/Entnahmevorgängen jeweils für $\mathrm{PbW}$ und $\mathrm{PbP}$ )

3. Bearbeitung von fünf Aufträgen mit einem der beiden AS

4. Ausfüllen eines Fragebogens zu Merkmalen der Teilnehmer und zur Beurteilung des zuerst genutzten Systems

5. Bearbeitung von fünf Aufträgen mit dem verbleibenden AS

6. Ausfüllen eines Fragebogens zur Beurteilung des anderen AS.

Der PbW-Prozess begann mit der Anmeldung des Teilnehmers an einem Leitstand mittels vorher ausgegebener Zugangsdaten. Danach konnte ein Proband mit einer ersten Wischbewegung auf der kreisförmigen Uhrenoberfläche (Bildschirmdiagonale: 3,5 cm) die Zuordnung eines Auftrags (Benennung der Gesamtzahl der zu abzuholenden verschiedenen Artikel) über das WLAN anstoßen (s. Abb. 1, links).

Mit einer zweiten Wischbewegung wurde für den ersten Artikel dessen Lagerplatz (Regalzeile, Regalebene, Fachnummer) angezeigt. Dort war ein dreistelliger alpha-

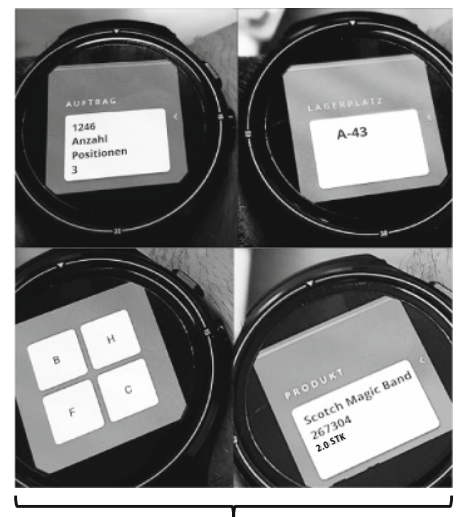

Pick-by-Watch-System

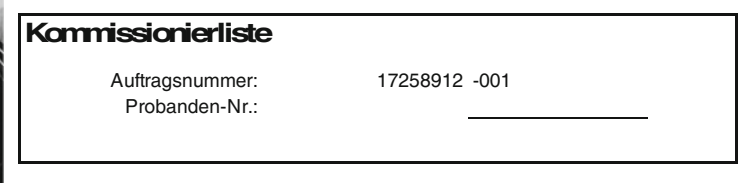

\begin{tabular}{|ccccccc|}
\hline Pos. & $\begin{array}{c}\text { Lagerplatz- } \\
\text { nummer }\end{array}$ & Menge & Enheit & Artikelnummer & Artikelbezeichnung Ablageort \\
\hline 1 & B-22 & 11 & Stck & $797639(\mathrm{Z})$ & Böhme Zitrone & KB \\
\hline 2 & C-44 & 3 & Stck & 5204 B2640G & Batterie AAA & KB \\
\hline 3 & D-46 & 14 & Stck & 797639 (O) & Böhme Orange & KB \\
\hline 4 & & & & & & \\
\hline 5 & & & & & \\
\hline
\end{tabular}

Pick-by-Paper-System

Abb. 1 Darstellung von Auftragsinformationen in den Assistenzsystemen 
betischer Code angebracht, der über die berührungsempfindliche Oberfläche der Uhr einzugeben war. Nach einer korrekten Codeangabe wurden auf der Uhr Artikelbezeichnung und -nummer sowie Entnahmemenge angezeigt. Bei einem falschen Code vibrierte die Uhr als Hinweis auf einen Fehler und Aufforderung zur erneuten Eingabe des korrekten Codes. Die entnommenen Exemplare eines Artikels wurden in einem Behälter auf dem mitgeführten Schiebewagen abgelegt. Danach forderte der Komissionierer mit einer zusätzlichen Wischbewegung auf der Oberfläche der Uhr Daten zum Lagerplatz des nächsten Artikels des Auftrags an. Artikel wurden solange auf der Uhr gezeigt bis der letzte Artikel eines Auftrags entnommen war und das Auftragsende in grün auf der Uhr mitgeteilt wurde. Dann kehrte der Proband mit dem Schiebewagen zum Startort zurück. Anschließend wurde dem Kommissionierer solange ein neuer Auftrag zugewiesen bis fünf Aufträge mit dem PbW-System erledigt waren.

Bei der PbP-Kommissionierung wurden pro Auftrag die Daten in Form einer durchnummerierten Liste auf einem DIN-A4-Blatt zur Verfügung gestellt (s. Abb. 1, rechts). Die zu bearbeitenden fünf Aufträge wurden hintereinander auf einem Klemmbrett befestigt, das dem Probanden übergeben wurde. Dabei wurde darauf hingewiesen, dass (1) ein Ablegen des Klemmbretts untersagt war, um auf eine einhändige Entnahme von Artikeln hinzuwirken, und (2) die Quittierung der Entnahme von Artikeln auf der Liste durch Setzen eines Zeichens gestattet, aber nicht zwingend gefordert war. Das Vorgehen beim Anlaufen der Artikel wurde dem Kommissionierer freigestellt, so dass er sich nicht an die Reihenfolge zu halten hatte, die auf der Papierliste angegeben war. Nach Entnahme sämtlicher Artikel eines Auftrags kehrte der Proband mit dem Schiebewagen zum Startpunkt zurück. Dort wurden die Artikel des Auftrags vom Wagen entfernt. Anschließend wurde der Schiebewagen erneut am Startpunkt positioniert und der nächste Auftrag abgearbeitet. Dieser Prozess wurde wiederholt bis fünf Aufträge bewältigt waren.

\subsection{Untersuchungsteilnehmer}

Zur Rekrutierung von Studienteilnehmern wurden in einem ersten Schritt an der Universität, an der die Verfasser tätig sind, Angestellte, Beamte und Studenten persönlich darum gebeten, durch Eintrag in eine Online-Terminliste ihre Bereitschaft zum Durchlaufen des Experimentallagers zu signalisieren. Die so gewonnenen Probanden wurden nach ihrer Mitwirkung an dem Experiment in einem zweiten Schritt aufgefordert, andere Personen auf die Untersuchung hinzuweisen und den Link zur Terminvereinbarung für eine Teilnahme an sie weiterzuleiten. Als Anreiz für eine Mitwirkung wurde die im Experiment eingesetzte Smart Watch unter den Teilnehmern verlost.

Insgesamt wurde so eine (Gelegenheits-)Stichprobe von 55 Personen akquiriert. Damit basieren die eigenen Analysen auf einem Stichprobenumfang, der zumeist doppelt so groß ist wie in vergleichbaren empirischen Studien zu AS in der Kommissionierung, von denen die Arbeit von Stockinger et al. (2020) mit 31 Teilnehmern nach unserem besten Wissen die größte Stichprobe aufweist. Tab. 1 profiliert das eigene Sample im Hinblick auf per schriftlicher Befragung gewonnene Angaben (s. Schritt 4 in Abschn. 2.1) zu drei sozio-demographischen Merkmalen (s. Varia- 
Tab. 1 Merkmale der Untersuchungsteilnehmer $(n=55)$

\begin{tabular}{lr}
\hline 1. Alter ${ }^{a}$ & \\
18-19 Jahre & $1,8 \%$ \\
20-29 Jahre & $60,0 \%$ \\
30-39 Jahre & $23,6 \%$ \\
40-49 Jahre & $7,3 \%$ \\
50-66 Jahre & $7,3 \%$ \\
2. Geschlecht & \\
Weiblich & $47,3 \%$ \\
Männlich & $52,7 \%$ \\
3. Nationalität & \\
Deutsch & $61,8 \%$ \\
Nicht deutsch & $38,2 \%$ \\
4. Kommissioniererfahrung & \\
Nein & $87,3 \%$ \\
Ja & $12,7 \%$ \\
5. Nutzungserfahrung Smart Watches & \\
Keine Erfahrung & $56,4 \%$ \\
Einige Erfahrung & $25,4 \%$ \\
Umfangreiche Erfahrung & $18,2 \%$ \\
\hline
\end{tabular}

${ }^{\mathrm{a}}$ Mittelwert 30,1 Jahre, Standardabweichung 10,6 Jahre, Median 26 Jahre

blen 1-3) sowie zwei Charakteristika, die Erfahrungsaspekte widerspiegeln (s. Variablen 4-5).

Das Alter der Teilnehmer bewegte sich zwischen 18 und 66 Jahren bei einem Mittelwert (Median) von 30,1 (26,0) Jahren. 47,3\% der Versuchspersonen waren Frauen. 50,9\% gaben an, ein (Fach-)Hochschulstudium abgeschlossen zu haben. $38,2 \%$ berichteten, nicht die deutsche Nationalität zu besitzen. Von den 21 Probanden ohne deutsche Staatsangehörigkeit hatten 13 die türkische sowie vier eine osteuropäische Nationalität, und die restlichen vier Personen verteilten sich auf drei weitere Länder. Im Vergleich zur Grundgesamtheit der volljährigen Bevölkerung in Deutschland sind damit ältere Personen ab 40 Jahre ohne Hochschulabschluss und mit deutscher Staatsangehörigkeit in unserer Stichprobe zu selten vertreten (vgl. Statistisches Bundesamt 2020).

$87,3 \%$ der Probanden beantworteten die Frage „Haben Sie bereits früher als Kommissionierer gearbeitet?“ mit „Nein“ (s. Variable 4). Der Anteil der Teilnehmer, die erklärten, keine Erfahrung mit Smart Watches zu haben, lag mit 56,4\% merklich niedriger (s. Variable 5). Ein Vergleich der Ausprägungen der zwei Erfahrungsmerkmale in unserer Stichprobe mit Werten für die erwachsene Gesamtbevölkerung in Deutschland ist aufgrund des Fehlens einschlägiger veröffentlichter amtlicher Statistiken nicht möglich. Indikativ lässt sich aber feststellen, dass (1) im eigenen Sample Personen mit umfangreicher Erfahrung beim Einsatz berührungsempfindlicher Smart Phone-Oberflächen angemessen erfasst sein sollten und (2) nicht ersichtlich ist, warum die geringe Kommissioniererfahrung in der Stichprobe nicht der Ausprägung dieser Variablen in der Grundgesamtheit entsprechen sollte. 


\subsection{Leistungsdimensionen}

Als Leistungsdimensionen wurden getrennt für jedes AS zum einen zwei Kriterien, die allein über Beobachtungen nicht-reaktiv erhoben wurden (tatsächliches Verhalten), und zum anderen drei Kriterien über Einschätzungen der Teilnehmer (erklärte Präferenzen) gemessen (s. Tab. 2).

In Anlehnung an frühere empirische Arbeiten zu AS in der Kommissionierung (z. B. Funk et al. 2016) wurde als erste objektive Dimension die Zeit (in Sekunden), die ein Kommissionierer vom Start der Bearbeitung eines Auftrags bis zur Rückkehr mit dem Schiebewagen zum Ausgangspunkt benötigte, gestoppt. Da sich Aufträge hinsichtlich der Zahl der Artikel und der pro Artikel zu entnehmenden Mengeneinheiten unterscheiden, wurde die Zeitvariable pro Auftrag normiert, indem die gestoppte Zeitdauer durch die pro auftragsrelevantem Artikel entnommenen Mengeneinheiten geteilt wurde. Anschließend wurde der Mittelwert der Zeit pro Mengeneinheit über alle fünf Aufträge berechnet (s. erster Ergebnisblock in Tab. 2). Der Mittelwert der PbW-Zeitdauer übersteigt mit 4,23 s ( $\mathrm{SD}=1,09)$ signifikant $(t=12,07$; $\mathrm{df}=54 ; p \leq 0,001)$ den PbP-Durchschnitt, der sich auf 3,17 s $(\mathrm{SD}=0,84)$ beläuft. Als zweite objektive Leistungsdimension wurde nach Abschluss der fünf Aufträge pro AS die Zahl jeglicher Kommissionierfehler (Mengen-, Auslassungs- und Typfehler; vgl. Günthner und Rammelmeier 2012) pro entnommener Mengeneinheit eines Auftrags sowie der Mittelwert der Fehler über die fünf Aufträge bestimmt (s. zweiter Ergebnisblock in Tab. 2). Der Mittelwert dieses Kriteriums beträgt für das PbWAS 0,009 $(\mathrm{SD}=0,008)$ und für das PbP-AS 0,007 $(\mathrm{SD}=0,008)$. Der Unterschied ist nicht auf dem 10\%-Niveau signifikant $(t=1,39$; $\mathrm{df}=54)$. Die intrapersonalen Korrelationen der beiden objektiven Kriterien sind mit $r=0,18$ für $\mathrm{PbW}$ und 0,23 für $\mathrm{PbP}$ jeweils nicht auf dem 5\%-Niveau statistisch signifikant (s. Tab. 4 im Anhang), d.h., dass höhere Geschwindigkeit nicht mit höherer Fehlerzahl „erkauft“ wurde.

Die drei subjektiven Leistungsdimensionen wurden getrennt für jedes AS mittels eines Fragebogens erhoben, den die Teilnehmer direkt nach Bearbeitungsende der fünf Aufträge für das unmittelbar zuvor genutzte System ausfüllten. Das erste Kriterium empfundene Beanspruchung umfasst den Durchschnitt der Antworten zu vier Items zu (aus objektiven Belastungen resultierenden) erlebten geistigen und körperlichen Beanspruchungen durch das verwendete AS (s. dritter Ergebnisblock in Tab. 2; vgl. zur konzeptionellen Einordnung dieser Beanspruchungsfelder Schlick et al. 2018, S. 266). Die Items (siehe Tab. 3 im Anhang) wurden der Erhebung von Bächler (2017) entnommen. Die interne Konsistenzreliabilität dieser Skala erreicht mit Cronbach $\alpha$-Werten von 0,74 für $\mathrm{PbW}$ und 0,77 für $\mathrm{PbP}$ ein gutes Niveau. Die Beanspruchung wird bei $\mathrm{PbW}$ im Durchschnitt als signifikant schwächer wahrgenommen als bei $\mathrm{PbP}(t=6,51 ; \mathrm{df}=54 ; p \leq 0,001 ;$ s. a. Fußnote a in Tab. 2$)$.

Die zweite subjektive Reaktionsdimension ist der ebenfalls in Anlehnung Bächler (2017) operationalisierte empfundene Zeitdruck bei der Auftragsbearbeitung mit einem AS (s. vierter Ergebnisblock in Tab. 2). Die Mittelwerte dieser Leistungsdimension unterscheiden sich zwischen PbW- und PbP-AS nicht signifikant $(t=1,05$; $\mathrm{df}=54 ; p \leq 0,297$; s. a. Fußnote $\mathrm{b}$ in Tab. 2).

Das dritte subjektive Leistungskriterium AS-Übungsaufwand gibt den Zustimmungsgrad der Probanden zu dem Item „Das Arbeiten mit dem Assistenzsystem er- 


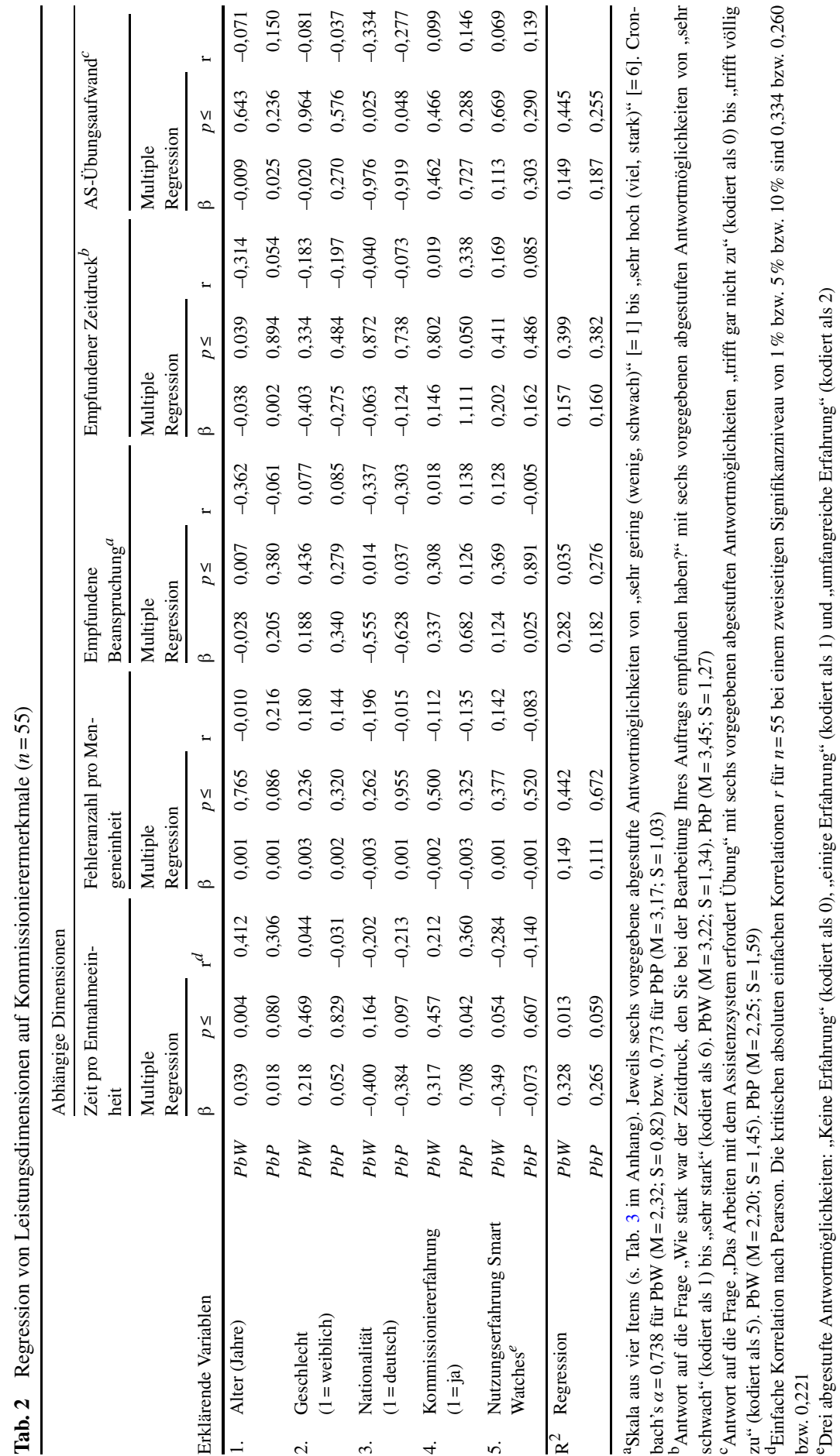


fordert Übung“" wieder (s. fünfter Ergebnisblock in Tab. 2). Der AS-Unterschied der Durchschnittswerte für dieses Leistungsmaß ist ebenfalls nicht signifikant $(t=0,22$; df $=54 ; p \leq 0,830 ;$ s. a. Fußnote $\mathrm{c}$ in Tab. 2).

Insgesamt weist somit in der Stichprobe das PbW-AS im Vergleich zum PbP-System bei einem objektiven Kriterium im Durchschnitt ein signifikant schlechteres und bei einem subjektiven Kriterium ein signifikant besseres Leistungsniveau auf. Bei drei Dimensionen sind die Mittelwerte ähnlich. Die subjektiven und objektiven Leistungsmessungen korrelieren überwiegend nicht signifikant (s. Tab. 4 im Anhang). Eine überraschende Ausnahme ist, dass bei PbW-Probanden mit unterdurchschnittlicher Kommissionierzeit stärkeren Zeitdruck empfinden, wohingegen im PbP-Fall schneller arbeitende Kommissionierer niedrigeren Zeitdruck wahrnehmen.

\section{Empirische Hypothesenprüfung}

Die sechs Hypothesen wurden mittels einfacher Korrelations- und multivariater Regressionsanalysen geprüft (s. Tab. 2).

Von den je fünf einfachen Korrelationen und $\beta$-Gewichten des Kommissionierermerkmals Alter sind für das PbW-AS je drei auf dem $5 \%$-Niveau signifikant: Höheres Alter geht einher mit geringerer Geschwindigkeit sowie niedrigeren Beanspruchungs- und Zeitdruckempfindungen. Erfolgt die Kommissionierung mit Papierlisten, dann sind keine signifikanten Zusammenhänge zu beobachten. Die Befunde stützen somit $\mathrm{H}_{1}$ und $\mathrm{H}_{2}$ nicht. Die Variable Geschlecht korreliert sowohl bei $\mathrm{PbP}$ als auch bei $\mathrm{PbW}$ mit keiner der Leistungsdimensionen in signifikanter Weise. Diese Ergebnisse bestätigen $\mathrm{H}_{3}$. Das Merkmal Nationalität weist signifikante Zusammenhänge mit zwei subjektiven Leistungskriterien im $\mathrm{PbW}$ - und im PbP-Fall auf: Kommissionierer ohne deutsche Nationalität empfinden die Beanspruchung durch Kommissionieraufgaben und den erforderlichen Übungsaufwand als signifikant höher als Probanden mit deutscher Staatsangehörigkeit. $\mathrm{H}_{4}$ wird somit als teilweise bestätigt. Hingegen geht frühere Kommissionierungserfahrung bei einem $\mathrm{PbW}$-AS nicht mit besseren Kommissionierungsleistungen und bei einem PbPAS mit langsamerer Aufgabenbewältigung sowie höherem wahrgenommenen Zeitdruck einher. Diese Resultate widersprechen $\mathrm{H}_{5}$. Allgemeine Nutzungserfahrungen mit Smart Watches senken im PbW-Fall die pro Entnahmeeinheit benötigte Zeit, korrelieren aber nicht signifikant mit den anderen vier Leistungsdimensionen. Erfolgt die Kommissionierung mit Papierlisten, sind keine Zusammenhänge zwischen allgemeinen Nutzungserfahrungen mit Smart Watches und den fünf Leistungsdimensionen festzustellen. Die Daten stützen also $\mathrm{H}_{6}$ für $\mathrm{PbW}$ eingeschränkt bei einem objektiven Leistungskriterium und für $\mathrm{PbP}$ uneingeschränkt.

\section{Fazit und Perspektiven}

Diese Arbeit berichtet Ergebnisse eines Laborexperiments mit der 55 Teilnehmern, die bei der auftragsbezogenen Entnahme von Gütern aus einem Lager durch ein $\mathrm{PbW}$ - oder ein PbP-AS unterstützt wurden. Schwerpunkt der Analyse sind Zu- 
sammenhänge zwischen fünf individuellen Merkmalen der Kommissionierer und fünf Leistungsindikatoren. Alles in allem sind nur zwischen der objektiv gemessenen Zeitdauer der Kommissionierung sowie der empfundenen Beanspruchung und den Personenmerkmalen Alter, Nationalität und allgemeine Nutzungserfahrung mit Smart Watches statistisch signifikante Zusammenhänge in dem Fall zu beobachten, dass ein $\mathrm{PbW}$-AS zum Einsatz kommt. Die untersuchten fünf individuellen Merkmale leisten demnach keinen herausragenden Beitrag zur Erklärung von Unterschieden im Hinblick auf Kommissionierungsleistungsapekte. Will man dennoch Personenmerkmale in der Einsatzpraxis nicht ganz außer Acht lassen, dann sollten sich nach unseren Resultaten (a) Einarbeitungsprogramme für PbW-AS bei knappen Ressourcen auf ältere Mitarbeiter mit Migrationshintergrund sowie ohne allgemeine Nutzungserfahrungen mit Smart Watches und (b) Rekrutierungsmaßnahmen auf Bewerber konzentrieren, die nicht dieses Merkmalsprofil aufweisen.

Der Befund, dass mit einem PbW-AS keine eindeutig besseren Kommissionierungsleistungen erzielt wurden als mit traditionellen Papierlisten deutet darauf hin, dass wichtige Hebel zur Steigerung von Kommissionierleistungen sämtlicher Aufgabenträger mehr bei der Gestaltung von PbW-AS selbst zu suchen sein dürften. Hier ist an Veränderungen der Displaygröße, -form und -auflösung, der Informationsdarstellung sowie der Rückmeldung auf Eingaben zu denken. PbW-AS erfordern - ähnlich wie Smart Glasses (s. Berkemeier et al. 2017, S. 791) - iterative Weiterentwicklungen und Leistungsmessungen, um ihre potenziellen Nutzenvorteile gegenüber ,altmodischen“ Papierlisten tatsächlich zu erschließen.

Für die weitere Forschung impliziert unsere Studie, dass es sinnvoll ist, objektive Produktivitätsmessungen mit subjektiven Leistungseinschätzungen für digitale AS in der Lagerhaltung zu kombinieren, um sie umfassend zu evaluieren. Da Erfahrungsaspekte in diesem Beitrag nur holzschnittartig erfasst wurden, liegt es außerdem nahe, in zukünftigen Arbeiten derartige Aspekte facettenreicher zu operationalisieren.

\section{Anhang}

Tab. 3 Fragen der Skala „Empfundene Beanspruchung“

Wie hoch waren die geistigen Anforderungen ${ }^{a}$ (z. B. Koordination, Konzentration, Kommunikation), die
dieses Assistenzsystem an Sie gestellt hat?
Wie viel körperliche Anstrengung war nötig, um dieses Assistenzsystem zu bedienen?
Wie stark mussten Sie sich insgesamt anstrengen, um den Kommissionierungsauftrag abzuarbeiten?
Wie stark fühlten Sie sich bei der Kommissionierung überfordert (Stressempfinden)?

${ }^{a}$ Fette Hervorhebungen entsprechen der optischen Darstellung im Fragebogen 


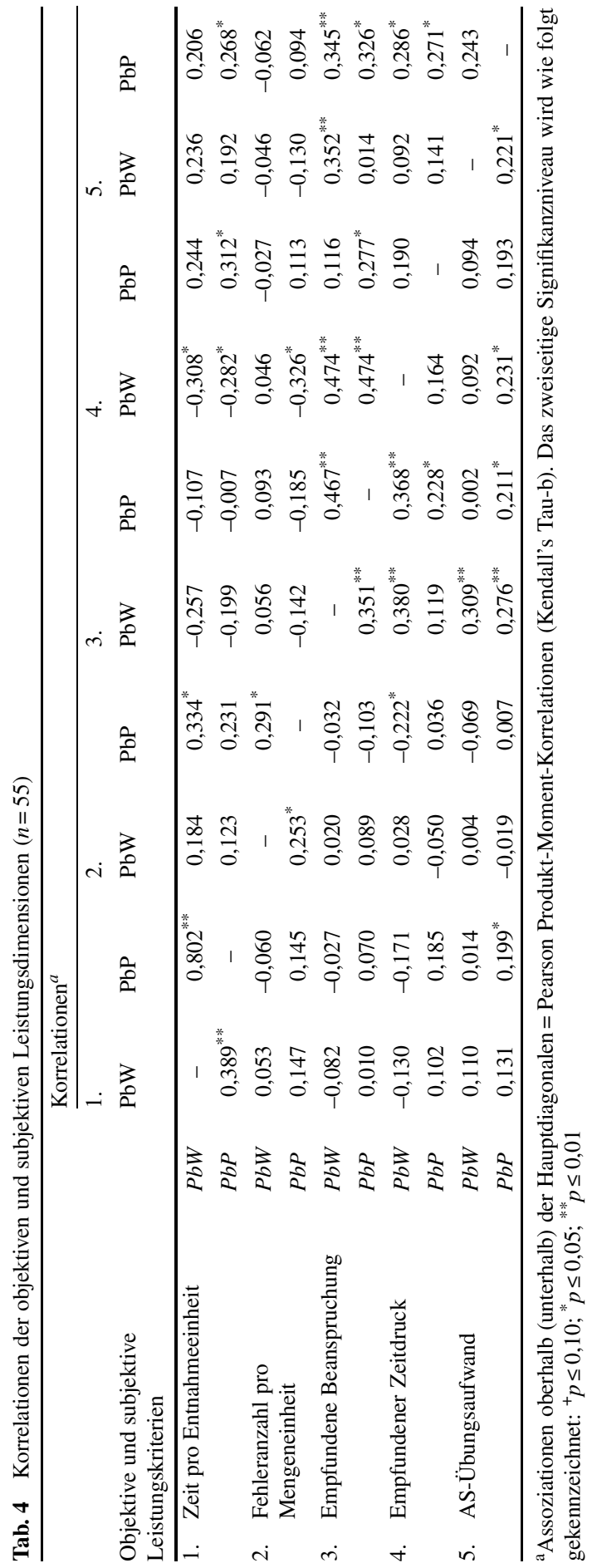


Funding Open Access funding enabled and organized by Projekt DEAL.

Open Access Dieser Artikel wird unter der Creative Commons Namensnennung 4.0 International Lizenz veröffentlicht, welche die Nutzung, Vervielfältigung, Bearbeitung, Verbreitung und Wiedergabe in jeglichem Medium und Format erlaubt, sofern Sie den/die ursprünglichen Autor(en) und die Quelle ordnungsgemäß nennen, einen Link zur Creative Commons Lizenz beifügen und angeben, ob Änderungen vorgenommen wurden.

Die in diesem Artikel enthaltenen Bilder und sonstiges Drittmaterial unterliegen ebenfalls der genannten Creative Commons Lizenz, sofern sich aus der Abbildungslegende nichts anderes ergibt. Sofern das betreffende Material nicht unter der genannten Creative Commons Lizenz steht und die betreffende Handlung nicht nach gesetzlichen Vorschriften erlaubt ist, ist für die oben aufgeführten Weiterverwendungen des Materials die Einwilligung des jeweiligen Rechteinhabers einzuholen.

Weitere Details zur Lizenz entnehmen Sie bitte der Lizenzinformation auf http://creativecommons.org/ licenses/by/4.0/deed.de.

\section{Literatur}

Bächler A (2017) Entwicklung und Evaluierung eines nutzerzentrierten Assistenzsystems zur Unterstützung von leistungsgeminderten Mitarbeitern bei manuellen Kommissioniertätigkeiten. Dissertation. Technische Universität, Ilmenau

Berkemeier L, Werning S, Zobel B, Ickerott I, Thomas O (2017) Der Kunde als Dienstleister: Akzeptanz und Gebrauchstauglichkeit von Smart Glasses im Self-Service. HMD 54(5):781-794

Diehl C, Hunkler C, Kristen C (2016) Ethnische Ungleichheiten im Bildungsverlauf. Eine Einführung. In: Diehl C, Hunkler C, Kristen C (Hrsg) Ethnische Ungleichheiten im Bildungsverlauf. Mechanismen, Befunde, Debatten. Springer, Wiesbaden, S 3-31

Egbert L, Quandt M, Thoben KD, Freitag M (2020) Mobile AR-based assistance systems for order picking-Methodical decision support in the early phases of the product life cycle. In: Freitag M, Kinra A, Kotzab H, Kreowski HJ, Thoben KD (Hrsg) Subject-oriented business process management. The digital Workplace-nucleus of transformation. S-BPM ONE 2020. Communications in Computer and Information Science, Bd. 1278. Springer, Cham, S 74-87 https://doi.org/10.1007/9783-030-64351-5_6

Esser H (2008) Assimilation, ethnische Schichtung oder selektive Akkulturation? Neuere Theorien der Eingliederung von Migranten und das Modell der intergenerationalen Integration. In: Kalter F (Hrsg) Migration, Integration und ethnische Grenzziehungen. Kölner Zeitschrift für Soziologie und Sozialpsychologie, Sonderheft, Bd. 48, S 81-107

Franzke T (2018) Der Mensch als Faktor in der manuellen Kommissionierung: Eine simulationsbasierte Analyse der Effizienz in Person-zur-Ware-Kommissioniersystemen. Springer, Wiesbaden

Funk M, Mayer S, Nistor M, Schmidt A (2016) Mobile in-situ pick-by-vision: Order picking support using a projector helmet. In: Proceedings of the 9th ACM International Conference on Pervasive Technologies Related to Assistive Environments. ACM, Corfu, S 45-48 https://doi.org/10.1145/2910674. 2910730

Gerpott TJ, Kurt A (2020) Assistenzsysteme in der Kommissionierung. Wirtschaftswissenschaftliches Stud 49(6):17-23

Van Gils T, Ramaekers K, Caris A, de Koster RB (2018) Designing efficient order picking systems by combining planning problems: State-of-the-art classification and review. Eur J Oper Res 267(1):1-15

Gogolin I, Klinger T, Schnoor B, Usanova I (2021) Mehrsprachigkeit an der Schwelle zum Beruf. Die Funktion sprachlicher Fähigkeiten für Berufsqualifizierung und Berufseinmündung von Jugendlichen mit und ohne Migrationshintergrund. Forschungsbericht, Universität Hamburg. https://www. pedocs.de/volltexte/2021/22047/pdf/Gogolin_et_al_2021_Mehrsprachigkeit_an_der_Schwelle.pdf. Zugegriffen: 28. Mai 2021

Grosse EH, Glock CH (2013) An experimental investigation of learning effects in order picking systems. J Manuf Technol Manag 24(6):850-872

Grosse EH, Glock CH (2015) The effect of worker learning on manual order picking processes. Int J Prod Econ 170(PC):882-890 
Grosse EH, Glock CH, Jaber MY, Neumann WP (2015) Incorporating human factors in order picking planning models: Framework and research opportunities. Int J Prod Res 53(3):695-717

Günthner WA, Rammelmeier T (2012) Auf dem Weg zur Null-Fehler-Kommissionierung. Neu entwickeltes Konzept mit Zukunftspotenzial. f+h Projektguide Intralogistik 2012 (Sonderausgabe), S 16-18

Hyde JS (2005) The gender similarities hypothesis. Am Psychol 60(6):581-592

Hyde JS (2014) Gender similarities and differences. Annu Rev Psychol 65(1):373-398

Ponis ST, Plakas G, Agalianos K, Aretoulaki E, Gayialis SP, Andrianopoulos A (2020) Augmented reality and gamification to increase productivity and job satisfaction in the warehouse of the future. Proc Manuf 51:1621-1628

Rhodes S, Greene NR, Naveh-Benjamin M (2019) Age-related differences in recall and recognition: A meta-analysis. Psychon Bull Rev 26(5):1529-1547

Schlick C, Bruder R, Luczak H (2018) Arbeitswissenschaft, 4. Aufl. Springer Vieweg, Berlin

Schunck R, Teltemann J (2019) Kompetenzungleichheit zwischen SchülerInnen mit und ohne Migrationshintergrund im Zeitvergleich. Informationsd Soz Indik 61(1):6-11

Seeber S, Busse R, Michaelis C, Baethge M (2019) Migration in der beruflichen Bildung: Herausforderungen für die Integration von zugewanderten schutz- und asylsuchenden Jugendlichen. Z Erziehungswiss 22(1):71-99

Siddiq F, Scherer R (2019) Is there a gender gap? A meta-analysis of the gender differences in students' ICT literacy. Educ Res Rev 27(1):205-217

Statistisches Bundesamt (2020) Bevölkerung in Privathaushalten nach Migrationshintergrund und höchstem allgemeinem Schulabschluss (höchstem beruflichem Bildungsabschluss, Altersgruppen). Statistisches Bundesamt, Wiesbaden. https:/www.destatis.de/DE/Themen/Gesellschaft-Umwelt/ Bevoelkerung/Migration-Integration/Tabellen/migrationshintergrund-schulabschluss.html. Zugegriffen: 28. Mai 2021 (https://www.destatis.de/DE/Themen/Gesellschaft-Umwelt/Bevoelkerung/ Migration-Integration/Tabellen/migrationshintergrund-beruflicherabschluss.html, https://www.desta tis.de/DE/Themen/Gesellschaft-Umwelt/Bevoelkerung/Migration-Integration/Tabellen/migrationshin tergrund-alter.html)

Stockinger C, Steinebach T, Petrat D, Bruns R, Zöller I (2020) The effect of pick-by-light-systems on situation awareness in order picking activities. Proc Manuf 45(1):96-101

Ten Hompel M, Sadowsky V, Mühlenbrock S (2019) Kommissioniersysteme. In: Schmidt T (Hrsg) Innerbetriebliche Logistik. Springer Vieweg, Berlin, S 113-152

Tripp C (2019) Distributions- und Handelslogistik. Springer, Wiesbaden

Tucker-Drob EM, Brandmaier AM, Lindenberger U (2019) Coupled cognitive changes in adulthood: A meta-analysis. Psychol Bull 145(3):273-301

VDI (1994) VDI 3590 Blatt 1: Kommissioniersysteme - Grundlagen. VDI-Gesellschaft Fördertechnik Materialfluss Logistik, Berlin

De Vries J, de Koster R, Stam D (2016) Exploring the role of picker personality in predicting picking performance with pick by voice, pick to light and RF-terminal picking. Int J Prod Res 54(8):2260-2274

Werning S, Berkemeier L, Zobel B, Fitte C, Ickerott I, Thomas O (2019) Smart Glasses als Assistenzsystem in der betrieblichen Einarbeitung. HMD 56(3):612-627 